\title{
Engineering Bio-compatible Interfaces via Combinations of Oxide Films and Organic Self-Assembled Monolayers
}

Xiaobo Yuan*, Nikolaus Wolf, Timm J. J. Hondrich, Pegah Shokoohimehr, Frano Milos, Manuel Glass, Dirk Mayer, Vanessa Maybeck, Michael Prömpers, Andreas Offenhäusser and Roger Wördenweber

Institute of Complex Systems - Bioelectronics (ICS-8), Forschungszentrum Jülich, Jülich 52428, Germany

*E-mail: x.yuan@fz-juelich.de

Tantalum pentoxide films with a typical thickness of $30 \mathrm{~nm}$ were deposited on the $90 \mathrm{~nm}$ thick $\mathrm{SiO}_{2}$ by atomic layer deposition (ALD) (FlexAl, Oxford Plasma Technology) using the corresponding precursors. Tris (diethylamido)(tert-butylimido) tantalum(V) (99.99\%) from Sigma-Aldrich (St. Louis) was used for the deposition of $\mathrm{Ta}_{2} \mathrm{O}_{5}$, yielding a growth rate of $0.081 \mathrm{~nm} /$ cycle at a substrate temperature of $300{ }^{\circ} \mathrm{C}$. The resulting films were examined via X-ray photoelectron spectroscopy (XPS), X-ray diffraction (XRD), Rutherford backscattering spectrometry (RBS) and scanning electron microscopy (SEM) in order to control the quality of the films. Examples of the characterization are summarized for $\mathrm{Ta}_{2} \mathrm{O}_{5}$ in Figure $\mathrm{S} 1 \mathrm{a}$ and Figure S1b.
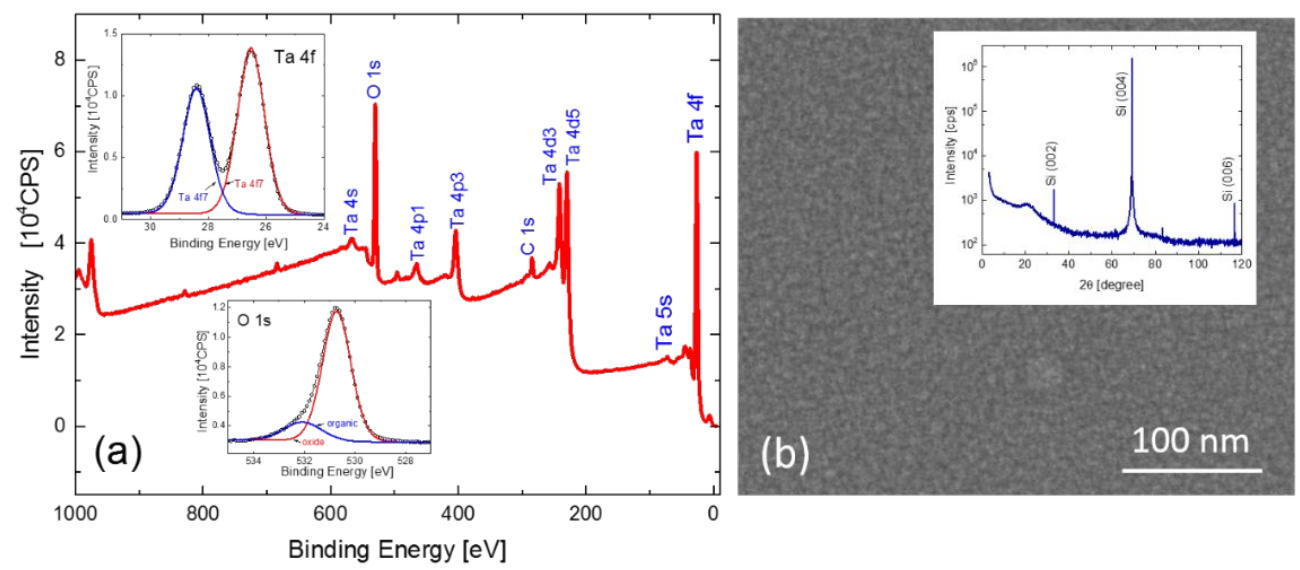

Figure S1. (a) XPS spectra depicting the ALD $\mathrm{Ta}_{2} \mathrm{O}_{5}$ specimen. Top insert and bottom insert show detailed XPS spectra scanning of Ta $4 \mathrm{f}$ and $\mathrm{O}$ 1s respectively. (b) SEM image of the surface morphology of the ALD $\mathrm{Ta}_{2} \mathrm{O}_{5}$; the insert shows the corresponding XRD BraggBrentano pattern.

XPS is used to analyze the surface chemical composition. The complete spectrum (0-1000 $\mathrm{eV}$ ) in Figure $\mathrm{S} 1 \mathrm{a}$ demonstrates the presence of $\mathrm{Ta}, \mathrm{O}$, and $\mathrm{C}$ at the surface. The analysis of the Ta 4f and O1s peaks (inserts in Figure S1a) demonstrates the following:

(i) Only one Ta-O phase is present at the surface. 
(ii) The $\mathrm{O} / \mathrm{Ta}$ ratio of $\sim 2.4$ obtained from the fitting of the spectra indicates that this phase is the $\mathrm{Ta}_{2} \mathrm{O}_{5}$ phase.

(iii) Although $\mathrm{C}$ 1s signals are unavoidable due to carbon contamination of ex situ prepared samples and XPS chamber components, it can't be excluded that at least parts of the $\mathrm{C}$ stems from organic hydrocarbon contaminations with $-\mathrm{OH},-\mathrm{C}=\mathrm{O}$, and $-\mathrm{COOH}$ groups might be the residue from the ALD precursor. RBS measurements support this interpretation and also show a contamination of $\sim 7.5$ atm $\%$ carbon in the films.

Furthermore, XRD analysis (insert in Figure S1b) demonstrates the absence of any regular (crystalline) structure of the $\mathrm{Ta}_{2} \mathrm{O}_{5}$ film and according to the SEM image (Figure S1b) the film is quite smooth.

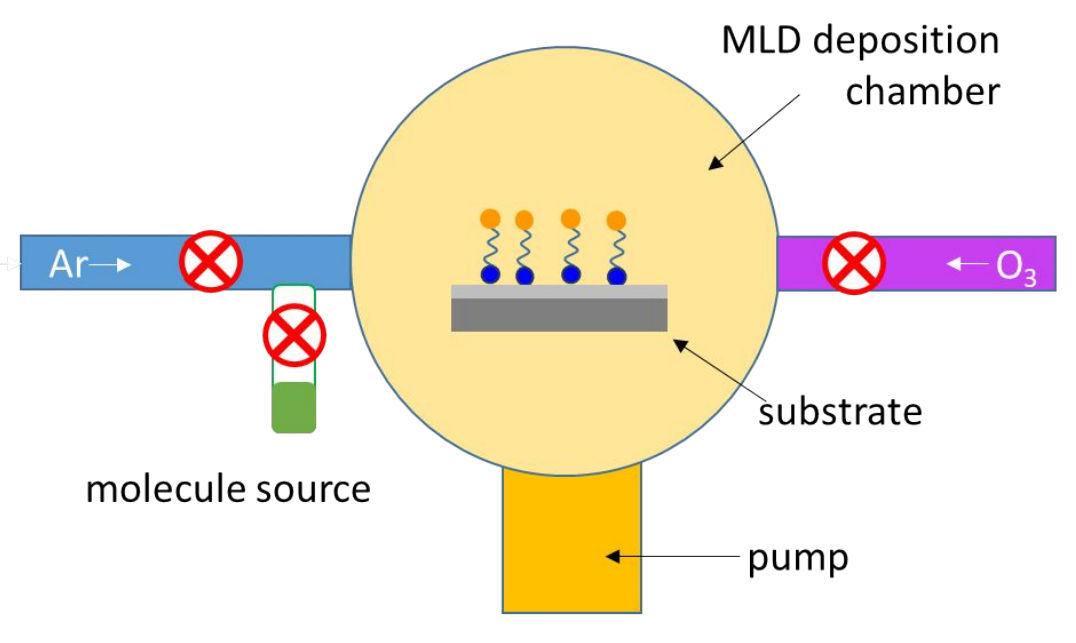

Figure S2: Diagram of the MLD setup, which shows (from left to right) the Ar gas supply (blue) for the working gas used during the deposition, the molecule source (green), the main chamber (yellow) including the in situ capacitive sensor - where activation and deposition takes place - and the ozone generator (purple). All essential ingredients are separated via valves (red) from the recipient, which is pumped with a turbo pump and an oil-free forepump. [1] 

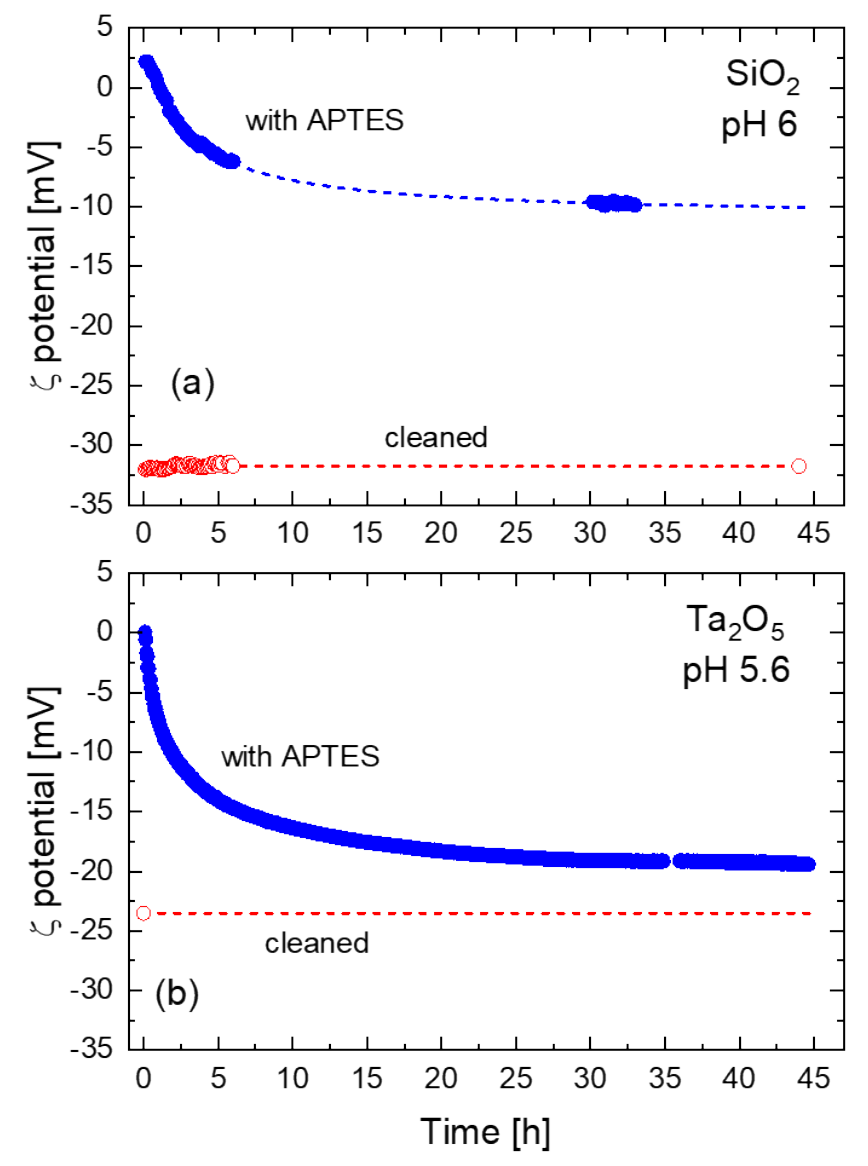

Figure S3. Comparison of the development of the $\zeta$ potential for (a) $\mathrm{SiO}_{2}$ and (b) $\mathrm{Ta}_{2} \mathrm{O}_{5}$ after coating with APTES (solid blue symbols) and cleaning (open red symbols) for streaming current measurement in a $1 \mathrm{mM} \mathrm{KCl}$ electrolyte at slightly different $\mathrm{pH}$ values. The APTES has been deposited at room temperature via gas-phase MLD using the standard parameters given in refs. [1,2] and a post-deposition time of $24 \mathrm{~h}$. Since the potential doesn't change for the cleaned state, the surface potential is extrapolated from a few data points.

The data in Figure S3a demonstrate that although a small amount of physisorbed APTES (positively charged functional group) is removed in the electrolyte flow, a stable chemisorbed APTES layer remains on the $\mathrm{SiO}_{2}$ surface which leads to a strongly enhanced surface potential. Additional characterization (e.g. contact angle measurements, fluorescence microscopy, ellipsometry; see refs. [1,2]) indicates that this layer is a chemically bound SAM. In contrast, the data in Figure S3b show that the majority of the APTES is removed in the electrolyte flow within the first two days. We conclude that APTES is not chemically bound to $\mathrm{Ta}_{2} \mathrm{O}_{5}$ and most of it is removed during the surface potential measurement. 


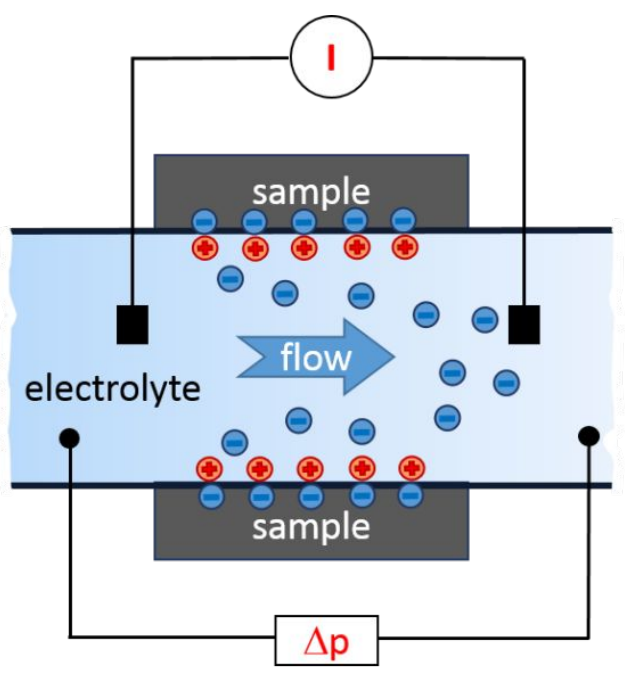

Figure S4: Schematic of the major component of the streaming current measurements setup consisting of a pair of identical planar substrates placed in a clamping cell with the surfaces to be analyzed facing each other and forming a microfluidic channel, as well as electrodes and sensors to determine the electric current as function of the flow of the electrolyte. ${ }^{[1]}$

(1) Yuan, X.; Wolf, N.; Mayer, D.; Offenhäusser, A.; Wördenweber, R. Vapor-Phase Deposition and Electronic Characterization of 3-Aminopropyltriethoxysilane Self-Assembled Monolayers on Silicon Dioxide. Langmuir 2019, 35, 8183-8190.

(2) Markov, A.; Wolf, N.; Yuan, X.; Mayer, D.; Maybeck, V.; Offenhäusser, A.; Wördenweber, R. Controlled Engineering of Oxide Surfaces for Bioelectronics Applications Using Organic Mixed Monolayers. ACS Appl. Mater. Interfaces 2017, 9, 29265-29272. 